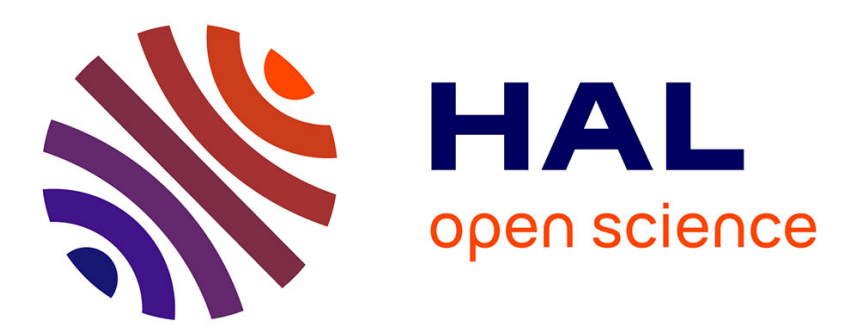

\title{
Global Stabilization with Low Computational Cost of the Discrete-Time Chain of Integrators by Means of Bounded Controls
}

Nicolas Marchand, Ahmad Hably, Ahmed Chemori

\section{- To cite this version:}

Nicolas Marchand, Ahmad Hably, Ahmed Chemori. Global Stabilization with Low Computational Cost of the Discrete-Time Chain of Integrators by Means of Bounded Controls. IEEE Transactions on Automatic Control, 2007, 52 (5), pp.948-952. 10.1109/TAC.2007.895956 . lirmm-00152310

\section{HAL Id: lirmm-00152310 \\ https://hal-lirmm.ccsd.cnrs.fr/lirmm-00152310}

Submitted on 6 Jun 2007

HAL is a multi-disciplinary open access archive for the deposit and dissemination of scientific research documents, whether they are published or not. The documents may come from teaching and research institutions in France or abroad, or from public or private research centers.
L'archive ouverte pluridisciplinaire HAL, est destinée au dépôt et à la diffusion de documents scientifiques de niveau recherche, publiés ou non, émanant des établissements d'enseignement et de recherche français ou étrangers, des laboratoires publics ou privés. 


\section{Global stabilization with low computational cost of the discrete-time chain of integrators by means of bounded controls}

\author{
Nicolas Marchand, Ahmad Hably, Student Member, IEEE, \\ and Ahmed Chemori
}

\begin{abstract}
The paper proposes a bounded nonlinear control law composed of saturation functions for the discrete time chain of integrators. A dynamical adaptation rule of the saturation levels involved in the control law is proposed to improve the closed-loop performances. The paper unifies the original work of Yang et al. (1997) with static saturation level and convergence improvements that recently appeared in the continuous time case. The possible ranges for the controller's parameters are extended with respect to existing results.
\end{abstract}

Index Terms-Bounded control, saturation functions, chain of integrators.

\section{INTRODUCTION}

Practical control applications obviously require bounded control in order to fit into the physical limits of the actuators (see for instance recent books [1], [2] or the special issue [3] and the chronological bibliography therein). Among the numerous existing methods, one can find the model predictive control (MPC). It is based on an online computation of an open-loop optimal input over a prediction horizon. The first step of the resulting optimal sequence is applied, and then the prediction horizon is shifted forward. The optimization problem is resolved again, and so on. MPC is a well-known method used for stabilizing linear systems with constrained control inputs [4], [5], [6], [7]. However, due to the intensive computations, this method is not always applicable to fast systems. Moreover, the optimal solution may be discontinuous as it is for the optimal time problem. The linear anti-windup compensation is widely used. The saturation effect is compensated by means of a linear feedback (see [8], [9] in continuous time and [10], [11] in discrete time). Unfortunately, as mentioned by Megretski [12], a rigorous stability and robustness analysis is hard to carry out. Low-gain control also gave rise to much literature [12], [13], [14], [15]. In this scheme, the saturation of a linear controller is usually obtained by solving a Riccati equation which depends on a specific parameter adapted online (without adaptation, only semiglobal stabilization is achieved) [13], [12], [16]. Unfortunately, in order to insure global asymptotic stability, a convex optimization problem must be solved at each time instant, a drawback that reduces the number of embedded and fast applications based on this type of control. Teel in [17] has proposed a nonlinear globally stabilizing control law composed of nested saturation functions for the continuous linear chain of integrators. Various works extended Teel's initial result to general controllable linear systems (in continuous time [18] or in discrete time [19]) and linear systems subject to measurement bounds [20]. The complexity of these methods is close to those for the unconstrained one. As mentioned in [21], a comparison paper for the double integrator case, the nonlinear approach shows good performance with respect to robustness and performance degradation. However, these nonlinear approaches suffer from slow convergence, especially for high dimensional systems.

N. Marchand and A. Hably are with the Control System Department of GIPSA-Lab, CNRS UMR 5216, ENSIEG BP 46, 38402 Saint Martin d'Hères Cedex, FRANCE. e-mail: \{Nicolas.Marchand, Ahmad. Hably\}einpg.fr

A. Chemori is with Laboratoire d'Informatique, de Robotique et de Microélectronique de Montpellier (LIRMM), UMR CNRS 5506, 161 rue Ada, 34392 Montpellier Cedex 05, FRANCE. e-mail: ahmed.chemorielirmm. fr
This problem was partially answered by two different approaches. Kaliora and Astolfi in [22] noticed (without testing it) that the state of the system can be weighted with a weight computed offline by means of a constrained optimization procedure. In [23], a dynamical adaptation of the saturation levels of the control law is proposed in function of the system's state. The simplicity is kept and the robustness properties are only slightly degraded. The convergence is sped up considerably compared with other methods and is no longer correlated to the system's dimension. Extending these results to the discrete time case is not trivial since owing to the saturation, the continuous time controller can not be discretized since it is nonlinear. The case with fixed saturations levels is treated in [19]. The purpose of the present paper is to enable dynamical adaptation of these levels in the discrete time case. In the proposed scheme, the conditions imposed on the parameters of the control law are less restrictive than in existing continuous time approaches. The control law unifies fixed and dynamic approaches and provides a parameter that enables to tune the degree of adaptation of the controller. As in the continuous time case, the control law shows good performances while keeping a very low computational complexity. In this paper, only the chain of integrators is considered, but the results can be extended to general linear systems following [19].

Notations: For any matrix $P, P_{i j}$ will stand for the element at the $i^{\text {th }}$ row and $j^{\text {th }}$ column, $P_{j}$ for its $j^{\text {th }}$ column. For any $y \in \mathbb{R}$, $\operatorname{sat}_{M}(y)=y$ if $|y| \leq M$ and $\operatorname{sat}_{M}(y)=\operatorname{sign}(y) M$ otherwise.

\section{PROBlem STATEMENT AND PRELIMINARY DEFINITIONS}

For continuous time systems, the chain of integrators of dimension $n$ is classically given by:

$$
\dot{x}=\mathcal{A} x+\mathcal{B} u, \quad x \in \mathbb{R}^{n}, u \in \mathbb{R}
$$

where

- $\mathcal{A}_{i j}=1$ for $i, j \in\{1, \ldots, n\}$ if $i+1=j$ and $\mathcal{A}_{i j}=0$ otherwise

- $\mathcal{B}_{i}=0$ for $i \in\{1, \ldots, n-1\}$ and $\mathcal{B}_{n}=1$.

For discrete time systems, two forms of the integrator chain are commonly considered. Both are treated in this paper. They are defined by

$$
x^{+}=A x+B u, \quad x \in \mathbb{R}^{n}, u \in \mathbb{R}
$$

where the pair $(A, B)$ can be either the Euler discretization of (1) with a normalized unitary sampling period:

- $\quad A_{i j}=1$ for $i, j \in\{1, \ldots, n\}$ if $i=j$ or $i+1=j$ and $A_{i j}=0$ otherwise

- $\quad B_{i}=0$ for $i \in\{1, \ldots, n-1\}$ and $B_{n}=1$.

or the $\mathrm{ZOH}$ discretization of (1) with some sampling period $T$ :

$$
\begin{array}{ll}
\text { - } & A_{i j}=\frac{T^{j-i}}{(j-i) !} \text { for } i, j \in\{1, \ldots, n\} \text { if } i \leq j \\
& \text { and } A_{i j}=0 \text { otherwise } \\
\text { - } & B_{i}=\frac{T^{n+1-i}}{(n+1-i) !} \text { for } i \in\{1, \ldots, n\} .
\end{array}
$$

The aim of this paper is to address the global stabilization of (2) with a control sequence $u$ that remains bounded by some a priori fixed level $\bar{u}$ :

$$
-\bar{u} \leq u \leq \bar{u}
$$

Similarly to the continuous time case [23], [18], [17], the system is first transformed into an appropriate state representation. On the contrary, the present proposed transformation is a little more general since the value of the parameters $\theta_{i}$ is not a priori fixed. 
Lemma 1: For any family of real numbers $\Theta=\left\{\theta_{i}\right\}_{i=1, \cdots, n}$, let $\left(A_{\Theta}, B_{\Theta}\right)$ be defined by:

$$
A_{\Theta}=\left(\begin{array}{ccccc}
1 & \theta_{2} & \theta_{3} & \ldots & \theta_{n} \\
0 & 1 & \theta_{3} & \ldots & \theta_{n} \\
\vdots & & \ddots & \ddots & \vdots \\
0 & \ldots & \ldots & 1 & \theta_{n} \\
0 & \ldots & \ldots & 0 & 1
\end{array}\right) \quad B_{\Theta}=\left(\begin{array}{c}
1 \\
1 \\
\vdots \\
1
\end{array}\right)
$$

Then, for any controllable pair $\left(A_{\Theta}, B_{\Theta}\right)$, there exists a coordinate change $y=P_{\Theta} x$ such that the system (2) becomes:

$$
y^{+}=A_{\Theta} y+B_{\Theta} u
$$

Note that $A_{\Theta}$ and $B_{\Theta}$ are independent from $\theta_{1}$. However, for the ease of the notation, the family $\Theta$ is defined with $n$ members that will all play a role in theorem 1 .

\section{Proof of Lemma 1: ${ }^{1}$}

Let $C$ and $C_{\Theta}$ be the controllability matrices of the pairs $(A, B)$ and $\left(A_{\Theta}, B_{\Theta}\right) . C$ and $C_{\Theta}$ are known to transform $(A, B)$ and $\left(A_{\Theta}, B_{\Theta}\right)$ into their canonical form that are identical since the eigenvalues of $A$ and $A_{\Theta}$ are the same. Hence, the transition matrix $P_{\Theta}$ is given by $C_{\Theta} C^{-1}$

\section{MAIN RESULTS}

The main result is formulated in the following theorem

Theorem 1: Let $\Theta:=\left\{\theta_{i}\right\}_{i=1, \cdots, n}$ be a family of $n$ real numbers such that:

- the pair $\left(A_{\Theta}, B_{\Theta}\right)$ as in (6) is controllable

- for all $k \in\{2, \ldots, n\}, 0<\sum_{i=1}^{k-1} \theta_{i}<\theta_{k}<1$

Let $\left\{M_{i}\right\}_{i=1, \ldots, n}$ be defined by:

$$
\left\{\begin{aligned}
M_{n}= & 1 \\
M_{j}= & 1+\alpha_{j} \frac{\theta_{j+1}}{\theta_{j}}\left[M_{j+1}-\left|\operatorname{sat}_{M_{j+1}}\left(\frac{y_{j+1}}{\sigma}\right)\right|\right], \\
& \text { for } j=1, \ldots, n-1
\end{aligned}\right.
$$

where $\alpha_{j} \in[0,1]$ for all $j=1, \ldots, n-1$ and $y=P_{\Theta} x$ with $P_{\Theta}$ as in Lemma 1. Let $\sigma:=\bar{u} / \sum_{i=1}^{n} \theta_{i}$, then, the control law

$$
u=-\sigma \sum_{i=1}^{n} \theta_{i} \operatorname{sat}_{M_{i}}\left(\frac{y_{i}}{\sigma}\right)
$$

globally asymptotically stabilizes the system (2) at the origin.

Theorem 1 is proved in the appendix A.

The $\alpha_{i}$ 's and the $\theta_{i}$ 's in the control law expression unifies the original works [18], [19] with the approaches that aim at improving the convergence [22], [23]. This enables to tune the degree of adaptation in relation with the robustness degradation allowed. Indeed, improving the convergence is paid with less robustness, in particular with respect to measurements delay.

$\alpha_{i}=0$ makes the level of the saturation independent of the state of the system. This approach was initially proposed in [18] for continuous time systems with $\theta_{i}$ 's restricted to $\theta_{i}<\epsilon^{n-i+1}$, $0<\epsilon \leq \frac{1}{2}$. The $\alpha_{i}$ 's enable to tune the degree of adaptation in relation with the robustness degradation allowed. Indeed, improving the convergence is paid with less robustness, in particular with respect to measurements delay [21], [23]. However, robustness properties remain acceptable for most applications when taking $\alpha_{i}=1$ that insure the fastest convergence of the states without introducing more computational complexity. Note that the convergence speed is known

\footnotetext{
${ }^{1}$ The authors thank anonymous reviewer for the suggestion of the proof
}

to be the main drawback of nested saturation approaches caused by an under utilization of the available input power in some state configuration [12]. As proposed in [22], the $\theta_{i}$ 's can be chosen using some optimization procedure constrained by the inequalities $\left\{0<\sum_{i=1}^{k-1} \theta_{i}<\theta_{k}<1\right\}_{k=2, \ldots, n}$ in order to insure the stability.

\section{Simulations}

Figure 1 shows the time evolution of system (1) when the control of Theorem 1 (taking $\theta_{i}=0.618^{n-i+1}$ ) is applied in a $\mathrm{ZOH}$ framework (that is applying $u$ constant between the time steps) for the initial condition $x_{0}=(2-23)^{T}$. Using the continuous time system (1) for the simulation instead of its discretization shows the behavior of the system between two time samples and enables the comparison with several other continuous time control approaches, that are the nonlinear saturated controls proposed in [17], [18] and [23], the minimum-time control (that corresponds to the best one can expect), and the low gain approach of Megretski [12] improved by [24]. The obtained performance shown in Figure 2 is very close to what can be expected using optimization with a computational cost similar to unconstrained control.
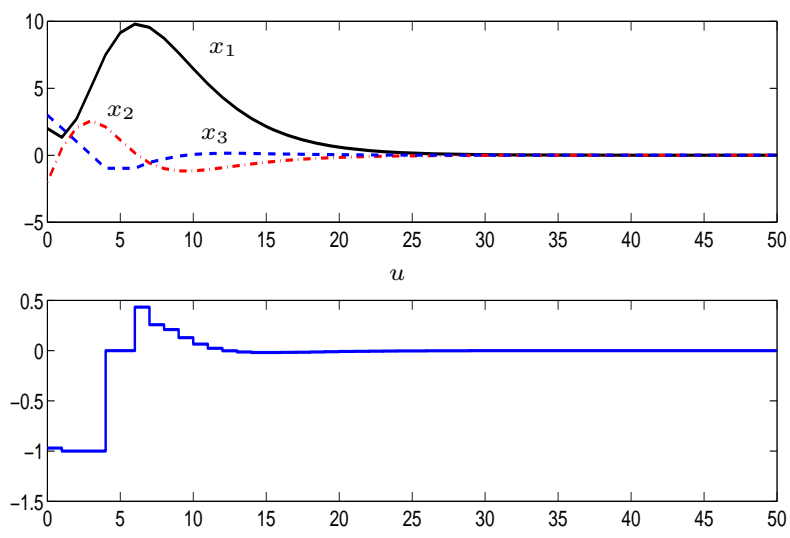

Fig. 1. Control of the third order integrator - Evolution of $x(t)$ and $u(t)$ with $T=1, x_{0}=(2-23)^{T}$ and $\theta_{i}=0.618^{n-i+1}$

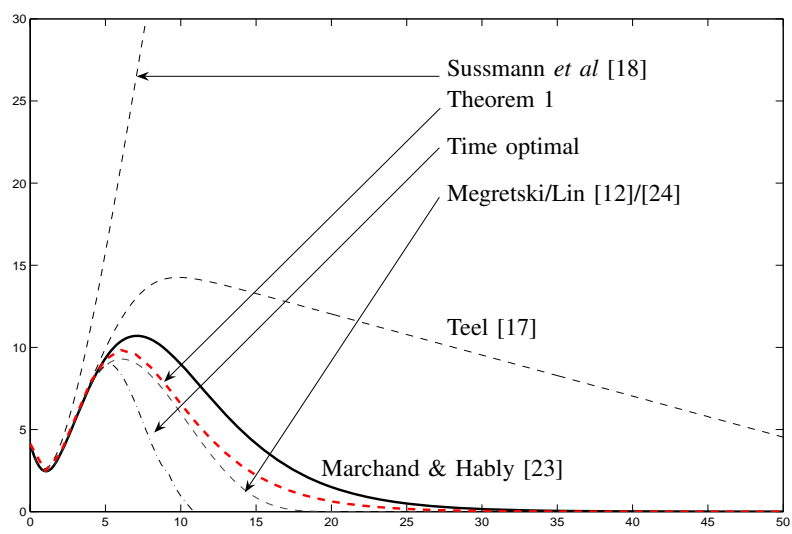

Fig. 2. Control of the third order integrator - Evolution of $\|x(t)\|$ for an initial condition $x_{0}=(2-23)^{T}$

\section{CONCLUSION}

In this paper, a bounded nonlinear control law composed of sum of saturation with possibly state dependent levels is proposed to stabilize the discrete time chain of integrators. The approach unifies 
the original work on this subject with recent improvements proposed in the literature in the continuous time case. The range for the control law parameters is increased. The approach combines a very low computational cost to competitive performances in terms of convergence speed.

\section{APPENDIX}

Let us consider the system (7) with $A_{\Theta}$ and $B_{\Theta}$ as in (6) and apply the coordinate change $z:=\frac{1}{\sigma} y$ and $v:=\frac{1}{\sigma} u$. The system (7) becomes:

$$
z^{+}=A_{\Theta} z+B_{\Theta} v
$$

with

$$
v=-\sum_{i=1}^{n} \theta_{i} \operatorname{sat}_{M_{i}}\left(z_{i}\right)
$$

Assume that $z_{n} \notin[-1,1]$. We will prove that $z_{n}$ necessarily joins $[-1,1]$ after a finite number of sampling time and remains therein after. Indeed, defining $V_{n}:=z_{n}^{2}$, it follows from (10):

$$
V_{n}^{+}-V_{n}=\left(z_{n}+v\right)^{2}-z_{n}^{2}=v^{2}+2 z_{n} v
$$

Clearly, thanks to the inequality $\theta_{n}>\sum_{i=1}^{n-1} \theta_{i}$ and the definition (11) of the control $v, z_{n} v$ is necessarily negative. Hence (12) can be rewritten:

$$
V_{n}^{+}-V_{n}=v^{2}-2\left|z_{n} v\right|
$$

With (11) one has:

$$
|v| \geq \theta_{n}\left|\operatorname{sat}_{M_{n}}\left(z_{n}\right)\right|-\sum_{i=1}^{n-1} \theta_{i}\left|\operatorname{sat}_{M_{i}}\left(z_{i}\right)\right|
$$

When $z_{n} \notin[-1,1],\left|\operatorname{sat}_{M_{n}}\left(z_{n}\right)\right|=1$, and therefore it follows using (8):

$$
\begin{aligned}
|v| \geq & \theta_{n}-\sum_{i=1}^{n-1} \theta_{i}\left|\operatorname{sat}_{M_{i}}\left(z_{i}\right)\right| \\
\geq & \theta_{n}-\sum_{i=2}^{n-1} \theta_{i}\left|\operatorname{sat}_{M_{i}}\left(z_{i}\right)\right|-\theta_{1} M_{1} \\
\geq & \theta_{n}-\sum_{i=2}^{n-1} \theta_{i}\left|\operatorname{sat}_{M_{i}}\left(z_{i}\right)\right| \\
& -\theta_{1}\left(1+\alpha_{1} \frac{\theta_{2}}{\theta_{1}}\left[M_{2}-\left|\operatorname{sat}_{M_{2}}\left(z_{2}\right)\right|\right]\right) \\
\geq & \theta_{n}-\theta_{1}-\sum_{i=3}^{n-1} \theta_{i}\left|\operatorname{sat}_{M_{i}}\left(z_{i}\right)\right| \\
& -\theta_{2} M_{2}+\theta_{2}\left(1-\alpha_{1}\right)\left[M_{2}-\left|\operatorname{sat}_{M_{2}}\left(z_{2}\right)\right|\right]
\end{aligned}
$$

since $\theta_{2}\left(1-\alpha_{1}\right)\left[M_{2}-\left|\operatorname{sat}_{M_{2}}\left(z_{2}\right)\right|\right] \geq 0$, it follows

$$
|v| \geq \theta_{n}-\theta_{1}-\sum_{i=3}^{n-1} \theta_{i}\left|\operatorname{sat}_{M_{i}}\left(z_{i}\right)\right|-\theta_{2} M_{2}
$$

Carrying on with the same reasoning, it gives:

$$
|v| \geq \theta_{n}-\sum_{i=1}^{n-1} \theta_{i}>0
$$

With the same reasoning, it also follows:

$$
\begin{aligned}
|v| & \leq \sum_{i=1}^{n} \theta_{i}\left|\operatorname{sat}_{M_{i}}\left(z_{i}\right)\right| \\
& \leq \sum_{i=2}^{n} \theta_{i}\left|\operatorname{sat}_{M_{i}}\left(z_{i}\right)\right|+\theta_{1}\left(1+\alpha_{1} \frac{\theta_{2}}{\theta_{1}}\left[M_{2}-\left|\operatorname{sat}_{M_{2}}\left(z_{2}\right)\right|\right]\right) \\
& \leq \theta_{1}+\sum_{i=3}^{n} \theta_{i}\left|\operatorname{sat}_{M_{i}}\left(z_{i}\right)\right|+\theta_{2} M_{2} \\
& \vdots \\
& \leq \sum_{i=1}^{n} \theta_{i}
\end{aligned}
$$

Since $\theta_{n}<1$, it gives:

$$
|v| \leq \theta_{n}+\sum_{i=1}^{n-1} \theta_{i} \leq 2-\theta_{n}+\sum_{i=1}^{n-1} \theta_{i}
$$

and therefore, since $z_{n} \notin[-1,1]$ :

$$
|v|-2\left|z_{n}\right| \leq-\theta_{n}+\sum_{i=1}^{n-1} \theta_{i}<0
$$

From inequalities (14) and (15), it follows in (13) that:

$$
V_{n}^{+}-V_{n} \leq-\left(\theta_{n}-\sum_{i=1}^{n-1} \theta_{i}\right)^{2}
$$

Hence, one can conclude that $V_{n}$ is strictly decreasing as long as $z_{n} \notin[-1,1]$ and $z_{n}$ necessarily joins $[-1,1]$ after a finite number of sampling period. Once $z_{n}$ has joined $[-1,1]$, one has:

$$
\left|z_{n}^{+}\right| \leq\left|\left(1-\theta_{n}\right) z_{n}\right|+\left|\sum_{i=1}^{n-1} \theta_{i} \operatorname{sat}_{M_{i}}\left(z_{i}\right)\right| \leq 1-\theta_{n}+\sum_{i=1}^{n-1} \theta_{i}<1
$$

Hence, once $z_{n}$ has joined $[-1,1]$, it remains therein for all future steps.

Assume now that $z_{n} \in[-1,1]$ and that $z_{n-1} \notin[-1,1]$. It is already known that $z_{n}$ remains in the interval $[-1,1]$, we shall prove that $z_{n-1}$ will also join this interval after a finite number of iterations. Indeed, defining $V_{n-1}:=z_{n-1}^{2}$, it follows from (10):

$$
\begin{aligned}
V_{n-1}^{+}-V_{n-1}= & \left(z_{n-1}+\theta_{n} z_{n}+v\right)^{2}-z_{n-1}^{2} \\
= & {\left[\sum_{i=1}^{n-1} \theta_{i} \operatorname{sat}_{M_{i}}\left(z_{i}\right)\right]^{2} } \\
& -2 z_{n-1} \sum_{i=1}^{n-1} \theta_{i} \operatorname{sat}_{M_{i}}\left(z_{i}\right)
\end{aligned}
$$

With similar arguments as for equations (14) and (15), one has:

$$
\left|\sum_{i=1}^{n-1} \theta_{i} \operatorname{sat}_{M_{i}}\left(z_{i}\right)\right| \geq \theta_{n-1}-\sum_{i=1}^{n-2} \theta_{i}>0
$$

and

$$
\left|\sum_{i=1}^{n-1} \theta_{i} \mathrm{sat}_{M_{i}}\left(z_{i}\right)\right| \leq \theta_{n-1}+\sum_{i=1}^{n-2} \theta_{i} \leq 2\left|z_{n-1}\right|-\theta_{n-1}+\sum_{i=1}^{n-2} \theta_{i}
$$

that gives in (16):

$$
V_{n-1}^{+}-V_{n-1} \leq-\left(\theta_{n-1}-\sum_{i=1}^{n-2} \theta_{i}\right)^{2}
$$


Hence, $z_{n-1}$ necessarily joins $[-1,1]$ after a finite number of time steps and $z_{n-1}^{+}$then fulfills:

$$
\begin{aligned}
\left|z_{n-1}^{+}\right| \leq & \left|\left(1-\theta_{n-1}\right) z_{n-1}\right|+ \\
& \left|\sum_{i=1}^{n-2} \theta_{i} \operatorname{sat}_{M_{i}}\left(z_{i}\right)\right| \leq 1-\theta_{n-1}+\sum_{i=1}^{n-2} \theta_{i}<1
\end{aligned}
$$

As a result, $z_{n+1}$ remains in the interval $[-1,1]$ for all future steps.

Continuing the same reasoning for all the $z_{i}$ 's, one can conclude that the state $z$ necessarily joins a unitary ball around the origin after a finite number of iterations. Once in the unitary ball, the control law (11) is linear and the closed loop system is given by:

$$
z^{+}=\left(\begin{array}{ccccc}
1-\theta_{1} & 0 & 0 & \cdots & 0 \\
-\theta_{1} & 1-\theta_{2} & 0 & \cdots & 0 \\
\vdots & \ddots & \ddots & & \vdots \\
\vdots & & \ddots & 1-\theta_{n-1} & 0 \\
-\theta_{1} & \ldots & & -\theta_{n-1} & 1-\theta_{n}
\end{array}\right) z
$$

that is obviously asymptotically stable since $\theta_{i}<1$ for all $i \in[1, n]$.

\section{REFERENCES}

[1] T. Hu and Z. Lin, Control systems with actuator saturation: Analysis and design. Birkhäuser, 2001.

[2] A. Saberi, J. Han, and A. A. Stoorvogel, Control of linear systems with regulation and input constraints, ser. Communications and Control Engineering. London, UK: Springer Verlag, 2000.

[3] D. S. Bernstein and A. N. Michel, Special issue on Saturating Actuators. Int. J. of Robust and Nonlinear Control, 1995, vol. 5.

[4] M. Athans and P. L. Falb, Optimal control. McGraw Hill, 1966.

[5] A. B. Lee and L. Markus, Foundations of optimal control theory. Wiley \& Sons, 1967.

[6] T. L. Vincent and W. J. Grantham, Nonlinear and optimal control. Wiley \& Sons, 1997.

[7] G. Goodwin, M. Seron, and J. De Dona, Constrained Control and Estimation - An Optimisation Approach, Springer, Ed., 2004.

[8] K. J. Åstrom and L. Rundquist, "Integrator windup and how to avoid it," in Proc. American Control Conference, 1989, pp. 1693-1698.

[9] P. J. Campo and M. Morari, "Robust control of processes subject to saturation nonlinearities," Computers and Chemical Engineering, vol. 14 , pp. $343-358,1990$.

[10] J. M. Gomes da Silva Jr. and S. Tarbouriech, "Local stabilization of discrete-time linear systems with saturating controls: an lmi-based approach," IEEE Trans. on Automatic Control, vol. 46, no. 1, pp. 119124, 2001.

[11] G. Grimm, J. Hatfield, I. Postlethwaite, A.-R. Teel, M.-C. Turner, and L. Zaccarian, "Antiwindup for stable linear systems with input saturation: an lmi-based synthesis," IEEE Transactions on Automatic Control, vol. 48, no. 9, pp. 1509-25, 2003.

[12] A. Megretski, " $\mathrm{L}_{2}$ BIBO output feedback stabilization with saturated control," in Proc. 13th IFAC World Congress, vol. D, 1996, pp. 435440.

[13] Z. Lin and A. Saberi, "Semiglobal exponential stabilisation of linear systems subject to input saturation via linear feedbacks," Systems \& Control Letters, vol. 21, no. 3, pp. 225-239, 1993.

[14] A. Saberi, Z. Lin, and A. R. Teel, "Control of linear systems with saturating actuators," IEEE Trans. on Automatic Control, vol. 41, no. 3, pp. 368-378, 1996.

[15] A. R. Teel, "Semi-global stabilizability of linear null controllable systems with input nonlinearities," IEEE Trans. on Automatic Control, vol. 40, no. 1, pp. 96-100, 1995.

[16] F. Grognard, R. Sepulchre, and G. Bastin, "Improved performance of low-gain designs for bounded control of linear systems," Automatica, vol. 38, no. 10, pp. 1777-1782, 2002.

[17] A. R. Teel, "Global stabilization and restricted tracking for multiple integrators with bounded controls," Systems \& Control Letters, vol. 18, no. 3, pp. 165-171, March 1992.

[18] H. J. Sussmann, E. D. Sontag, and Y. Yang, "A general result on the stabilization of linear systems using bounded controls," IEEE Trans. on Automatic Control, vol. 39, no. 12, pp. 2411-2425, Dec. 1994.
[19] J. Y. Yang, E. D. Sontag, and H. J. Sussmann, "Global stabilization of linear discrete-time systems with bounded feedback," Systems \& Control Letters, vol. 30, pp. 273-281, 1997.

[20] Z. Lin, "Global stabilization and restricted tracking for linear systems subject to input and measurement saturation-a chain of integrators case," in Proc. American Control Conference, vol. 4, 1995, pp. 2488-2492.

[21] V. G. Rao and D. S. Bernstein, "Naive control of the double integrator," IEEE Control Systems Magazine, vol. 21, pp. 86-97, Oct. 2001.

[22] G. Kaliora and A. Astolfi, "Nonlinear control of feedforward systems with bounded signals," IEEE Trans. on Automatic Control, vol. 49, no. 11, pp. 1975-1990, 2004.

[23] N. Marchand and A. Hably, "Global stabilization of multiple integrators with bounded controls," Automatica, vol. 41, no. 12, pp. 2147-2152, 2005.

[24] Z. Lin, "Global control of linear systems with saturating actuators," Automatica, vol. 34, no. 7, pp. 897-905, 1998. 